\title{
Preliminaryes studies of variability of intracranial pressure in military personnel undergoing combat tactics training in Brazilian air force
}

\begin{abstract}
The noninvasive intracranial pressure system can detect micrometric alterations in cranial volume. Alterations in the circulatory and respiratory systems, increased brain tissue, or problems in the production/reabsorption relationship of cerebrospinal fluid change the ICP auto regulation. The increase in pressure and blood flow during combat tactics can substantially increase intracranial pressure (ICP). The objective of this study was to evaluate and compare the ICP waveform characteristics in Military Personnel undergoing combat tactics training, in three different body positions: supine, seated and standing. Lysis of amplitude, heart rate, time between $\mathrm{P} 1$ and $\mathrm{P} 2$ peak, and $\mathrm{P} 1: \mathrm{P} 2$ ratio showed no statistical difference. This was also the case following morphological analysis of the ICP waveforms for the three different body positions. These initial findings form part of an ongoing, larger study. It can be observed that, although not statistically different, there is a tendency of a small variation in the variables analyzed, when ICP waveforms in the three body positions of the volunteers were compared.
\end{abstract}

\author{
Volume 2 Issue 5 - 2018
}

\author{
Thiago Augusto Rochetti Bezerra, ${ }^{1,2,7}$ Juliana \\ Cristina Viola, ${ }^{4}$ Phelipe Henrique Cardoso \\ de Castro, $2,3,7$ Edson Pereira Tangerino Filho, ${ }^{7}$ \\ Rodrigo Romero Faria Santos, ${ }^{2,6,7}$ Patrícia \\ Bezerra Lamego Alves, ${ }^{1,2,7}$ Áquila Denófrio \\ da Rocha, ${ }^{1,2,7}$ Grace Guindani ${ }^{1,5}$ \\ 'University of the Air Force, Post-Graduation Program in \\ Operational Human Performance/PPGDHO, Brazil \\ ${ }^{2}$ Physical Education Section of the Aeronautics Cadet Corps, Air \\ Force Academy, Brazil \\ ${ }^{3}$ Interstitutional Program of Post Graduation in Physiological \\ Sciences-PIPGCF UFSCar/UNESP, Brazil \\ ${ }^{4}$ Methodist University of Piracicaba-UNIMEP, Post-Graduation \\ Program in Human Movement Sciences, Brazil \\ ${ }^{5}$ Institute of Physical Activity Sciences-ICAF,Aeronautics Sports \\ Commission-CDA, Brazil \\ ${ }^{6}$ Faculty of Medicine of Ribeirão Preto, Postgraduate Program in \\ Health Sciences Applied to the Locomotor Apparatus, Brazil \\ ${ }^{7}$ Air Force Academy, Brazil
}

\begin{abstract}
Correspondence: Thiago Augusto Rochetti Bezerra, University of the Air Force, Post-Graduation Program in Operational Human Performance/PPGDHO, Rio de Janeiro, RJ,
\end{abstract} Brazil, Email thiago_rochetti@globomail.com

Received: August 06, 2018 | Published:September 10, 2018

\section{Introduction}

One of the factors that interfere with intracranial pressure (ICP) is blood pressure oscillation. Changes in blood pressure and redistribution of blood volume are caused by exposure to high intensity exercise and result from an increased hydrostatic gradient present in the venous and arterial systems. Therefore, evaluating individuals submitted to extreme physical effort can lead to an increase in ICP and this is consequently a branch of research that should be studied. An increase in the mean arterial pressure (MAP) leads to contraction of the vessels, until the value of $160 \mathrm{mmHg}$ is reached. From this limit the pressure causes passive dilatation in the vessels due to breakdown of the vascular resistance, and consequently cerebral blood flow elevation occurs (FSC). Cerebrospinal fluid (CSF) makes up 10\% of the inner volume of the cranial cavity. CSF is mainly produced in the choroid plexus of the cerebral ventricles at a rate ranging from 0.3 to $4 \mathrm{ml} / \mathrm{min}$.

The production of CSF, to a lesser extent (approximately 30\%), also occurs through the transudation of liquid by the ependyma. CSF reabsorption takes place predominantly in existing structures in the arachnoid membrane, called the arachnoid villi, a passive and unidirectional drainage system controlled by increased pressure in the cerebrospinal fluid. After production in the lateral ventricles, through the cerebral aqueduct, the CSF circulates through the foramina of Monro to the third ventricle and thence to the fourth ventricle through the cerebral aqueduct. From the fourth ventricle, the CSF leaves through the foramina of Luschka and Magendie and reaches the basal cisterns. Previously, through the anterior cisterns of the brainstem, the cerebrospinal fluid reaches the convexity of the brain after passing through the base of the frontal and temporal lobes. ${ }^{1,2}$ Later, the cerebrospinal fluid that comes out of the fourth ventricle circulates through the cisterna magna, supra cerebellar cisterns, ambient cisterns, and cisterns of the corpus callosum, reaching cerebral convexity. In addition, the CSF circulates around the medulla in the spinal canal, in an in and out movement in the cranial cavity. During exposure to muscle activity, an individual may be susceptible to differences in brain activation, with return of blood to the brain, and constant changes in blood pressure..$^{3-5}$ A study conducted with US military marines following a high-volume military training course, found that many military personnel had acute pain in the upper extremities, increased mean arterial pressure, and elevated serum muscle enzymes. Each student had followed a vigorous military training exercise program. The awareness of military leaders is necessary to avoid the potentially serious complication of renal failure and circulatory problems.

Biochemical data were collected from 19 soldiers who intermittently marched for 4 weeks, carrying approximately $45 \mathrm{~kg}$ of kit, with a limited intake of food and water. The mean serum creatine kinase activity was higher after gait, although the subjects did not develop 
symptomatic rhabdomyolysis. The level of serum osmolality did not present significant alterations. However, information on the variation in ICP in this type of military activity is still unknown. This study should thus bring unprecedented information, and with this contribute to better performance in exercises, emphasizing the importance of the study of intracranial pressure non-invasively. ${ }^{6-10}$ Evaluating the problems associated with variations in ICP could prevent further losses of the military, as well as improving combat and survival operational performance, reducing the social and financial losses in military institutions. The objective of this study was to monitor noninvasive intracranial pressure in infantry cadets under conditions of normality and after one week of training in Terrestrial Combat Tactics. ${ }^{4}$ The Ground Tactics Training (TTCT) course consisted of seven days of training. During this training the cadets performed practical combat activities. The daily distance covered was around $30 \mathrm{~km}$. The cadets carried the weight of their knapsack and armament with approximately $15 \mathrm{~kg}$ of equipment (Figure 1). Restrictions in sleep ( $2 \mathrm{~h}$ per day) and food, together with the large number of activities, mean that at the end of the week the personnel lose around 7 to $10 \mathrm{~kg}$ of body weight.

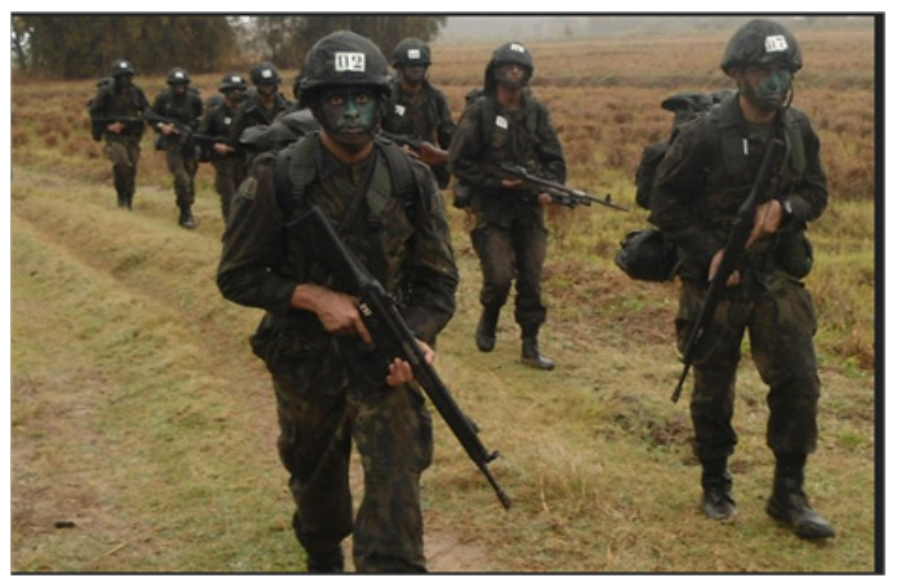

Figure I Infantry Cadets on Training Course of Terrestrial Combat Tactics I.

\section{Methodology}

For this work, 16 cadets of the Air Force Academy (AFA) were monitored in Pirassununga-SP-Brazil. The National Ethics Committee approved the project (CAAE: 40667114.7.0000.5504), and received financial support from the São Paulo Research Foundation (FAPESPProcess no. 2014/21803-7). The methodology used in this study was the use of non-invasive equipment for monitoring intracranial pressure (ICP). A Braincare Monitor BcMM-2000 (Braincare Health Technology) was used to monitor and record data with a sample rate of $200 \mathrm{~Hz}$. The intracranial pressure sensor used was the model BcSs1000 , developed by the Braincare team. The intracranial pressure of the volunteers was monitored alongside the temporal bone using a Braincare noninvasive intracranial pressure sensor. ${ }^{11,12}$ Data were collected before and after a week of ground combat tactics training in three different body positions: supine, seated and standing. The volunteers remained immobile during data collection. The data acquisition time was 5 (five) minutes. Ethical considerations were based on scientific purposes, with confidentiality of the infantry cadet's identity, free of coercion or conflict of interest of the institution or persons involved in the project. The cadets were previously informed and the ICP measured under express consent, provided using a specific form (Free and Informed Consent Term-TCLE), according to resolution 196/96 of CONEP. ${ }^{13-17}$
Data were acquired in an SD card and analyzed using algorithms developed in Python language for this study. ICP pulse wave morphology was isolated and the average of these pulses. The data analysis is composed by following processes:

a) Artifact identification.

b) Signal decomposition into trend and pulsatile components.

c) Pulse identification, separation and alignment.

d) Statistical analysis.

We first separate the artifact based on the windowed power spectral density ratio (SNR) between of the pulsatile component-frequencies between 0.5 and $3 \mathrm{~Hz}$-and raw signal up to $40 \mathrm{~Hz}$. We decomposed the signal into trend and pulsatile components by subtracting the DC component of the low pass signal (FIR filter with cutoff frequency of $20 \mathrm{~Hz}$ ). From the pulsatile component, we identify each pulse using the phase of the Hilbert transform. We perform second artifact elimination by identifying pulses that had amplitude or length $65 \%$ above or below the averages pulse properties. After pulse separation, we aligned iteratively all valid pulses using linear correlation between each pulse and mean pulse of period. The Kruskal-Wallis statistical test was applied, followed by the Mann-Whitney U test to compare the mean values between the three positions. Data are presented as mean \pm SEM, with a level of significance of $p<0.05$. We calculate the mean pulse properties-height, area, peaks positions, time to peak, length and inflection points-by local search of numerical maxima and minima on pulse waveform and its derivatives. Statistical inference on pulse properties was done using nonparametric statistical tests. All safety standards were adhered to in the study.

\section{Results}

The study cadet volunteers had the following characteristics (mean $\pm \mathrm{SEM}$ ): aged $21.3 \pm 0.27$ years, weight $72.8 \pm 1.75 \mathrm{~kg}$ and height $1.78 \pm 0.01 \mathrm{~m}$. It can be observed that the ICP pulse waveform did not change on either side of the temporal bone, where the sensor was positioned. The Figure 2 shows the ICP waveform being evaluated in three different body positions (supine, seated and standing) having 2 shows the ICP pulse waveform of 16 cadets. No difference was found in the ICP between the three positions until and after a week. Lysis of amplitude, heart rate, time between P1 and P2 peak, and P1:P2 ratio showed no statistical difference (Table 1). This was also the case following morphological analysis of the ICP waveforms for the three different body positions. It can be in Figure 3 we observed that there was no difference between the positions. In this part of the study, we menstruate the ICP in the right and left side of the temporal bone in the volunteers. Heartbeat as a function of position, for the right sensor A $(H=37.38, p<0.01)$ and left sensor B $(H=39.44, p<0.01)$. Sample size of 16 cadets. The red bars represent nonparametric confidence intervals $(\mathrm{a}=0.05, \mathrm{~N}=1000)$.

Table I Mean ( \pm SEM) ICP waveform values

\begin{tabular}{llll}
\hline & Supine & Seated & Standing \\
\hline Amplitude PI $(\mathrm{mV})$ & $0.03 \pm 0.01$ & $0.06 \pm 0.02$ & $0.04 \pm 0.01$ \\
$\mathrm{PI}-\mathrm{PI}(\mathrm{HR}, \mathrm{bPm})$ & $48 \pm 5$ & $54 \pm 6$ & $42 \pm 5$ \\
$\mathrm{PI}-\mathrm{P2}(\mathrm{s})$ & $0.17 \pm 0.02$ & $0.18 \pm 0.01$ & $0.11 \pm 0.01$ \\
$\mathrm{PI} / \mathrm{P2}$ & $3.18 \pm 1.4$ & $1.89 \pm 0.3$ & $2.09 \pm 0.60$ \\
\hline
\end{tabular}

$(n=5, \alpha=0.05)$ 


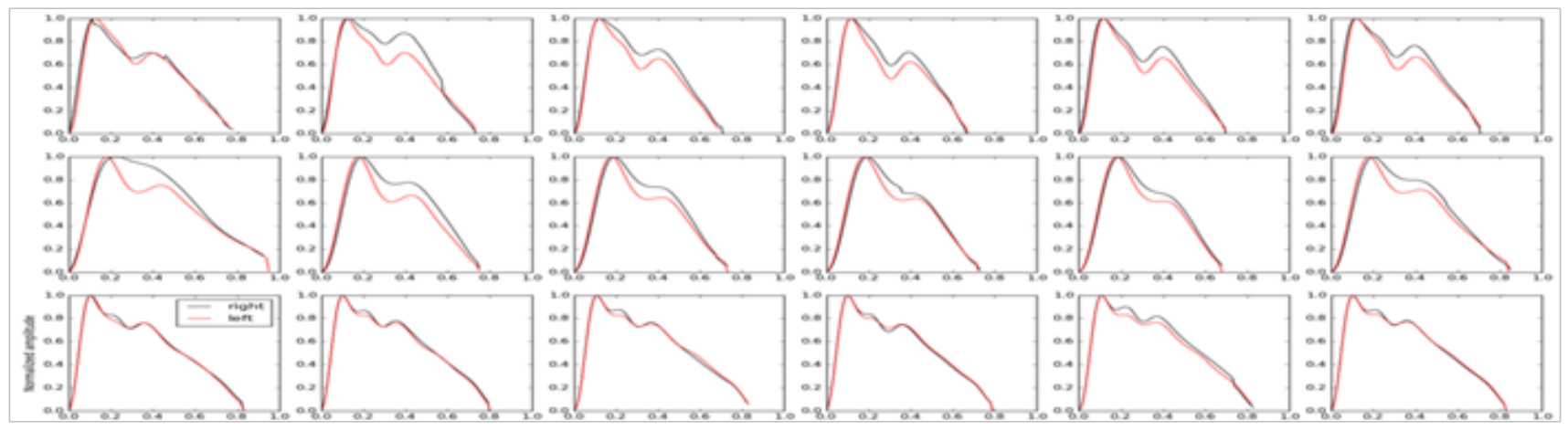

Figure 2 ICP waveform being evaluated in three different body positions (supine, seated and standing).
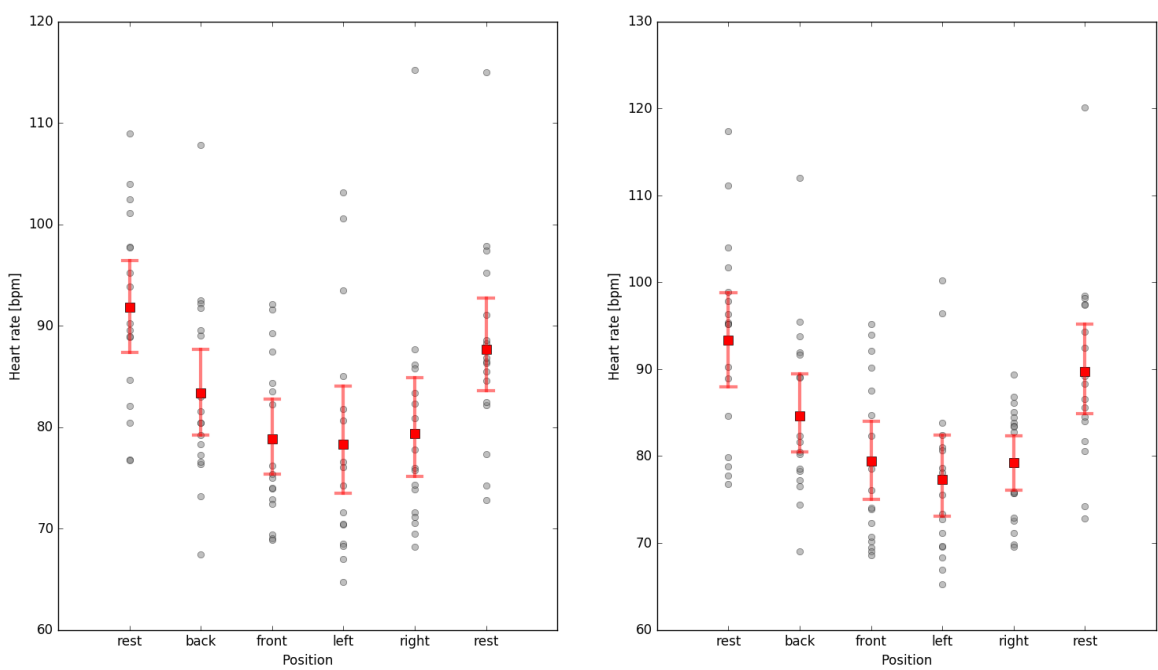

Figure 3 Heartbeat as a function of the position, for the right sensor $A(H=37.38, p<0.0 I)$ and left sensor $B(H=39.44, p<0.0 I)$.

\section{Conclusion}

These initial findings form part of an ongoing, larger study. It can be observed that, although not statistically different, there is a tendency of a small variation in the variables analyzed, when ICP waveforms in the three body positions of the volunteers were compared. The benefits to be found by this research are not limited. Numerous medical benefits can also be purchased. The data found can increase the central nervous system tolerance to ischemic hypoxia, present results directly linked to neurological diseases (stroke) and cardiovascular disease (heart attacks and sudden death).

\section{Future studies}

Further research is to be conducted involving the measurement of ICP in combination with arterial blood pressure. Subsequently, cadet will be monitored and measurements acquired during another's combat test.

\section{Acknowledgements}

None.

\section{Conflict of interest}

The author declares that they have no competing interests.

\section{References}

1. Schrouff J, Perlbarg V, Boly M, et al. Brain functional integration decreases during propofol-induced loss of consciousness. Neuroimage. 2011;57(1):198-205.

2. Lazow SP, Thadani VM, Gilbert KL, et al. Outcome of frontal lobe epilepsy surgery. Epilepsia. 2012;53(10):1746-1755.

3. Kim E, Lim YJ, Park HS, et al. The lack of relationship between intracranial pressure and cerebral ventricle indices based on brain computed tomography in patients undergoing ventriculoperitoneal shunt. Acta Neurochir. 2015;157(2):257-263.

4. Lang EW, Lagopoulos J, Griffith J, et al. Cerebral vasomotor reactivity testing in head injury: the link between pressure and flow. $J$ Neurol Neurosurg Psychiatry. 2003;74(8):1053-1059.

5. Koivisto AM, Kurki MI, Alafuzoff I, et al. High Risk of Dementia in Ventricular Enlargement with Normal Pressure Hydrocephalus Related Symptoms. J Alzheimer's Dis. 2016;52(2):497-507. 
6. Malm J, Graff-Radford NR, Ishikawa M, et al. Influence of comorbidities in idiopathic normal pressure hydrocephalus-research and clinical care. A report of the ISHCSF task force on comorbidities in INPH. Fluids Barriers CNS. 2013;10(1):22

7. Mori E, Ishikawa M, Kato T, et al. Guidelines for management of idiopathic normal pressure hydrocephalus: second edition. Neurol Med Chir (Tokyo). 2012;52(11):775-809.

8. Andresen M, Juhler M, Munch TN. Quality and safety of home ICP monitoring compared with in-hospital monitoring. Acta Neurochir Suppl. 2012;113:187-191.

9. Kristiansson H, Nissborg E, Bartek J, et al. Measuring elevated intracranial pressure through noninvasive methods: a review of the literature. J Neurosurg Anesthesiol. 2013;25(4):372-385.

10. Kohler BA, Ward E, McCarthy BJ, et al. Annual report to the nation on the status of cancer, 1975-2007, featuring tumors of the brain and other nervous system. J Natl Cancer Inst. 2011;103(9):714-736.

11. Winkler EA, Minter D, Yue JK, et al. Cerebral Edema in Traumatic
Brain Injury: Pathophysiology and Prospective Therapeutic Targets. Neurosurgery Clinics of North America. 2016;27(4):473-488.

12. Brown JA, Elliott MJ, Sray WA. Exercise-induced upper extremity rhabdomyolysis and myoglobinuria in shipboard military personnel. Mil Med. 1994;159(7):473-475.

13. Aizawa H, Morita K, Minami H, et al. Exertional rhabdomyolysis as a result of strenuous military training. J Neurol Sci. 1995;132(2):239-240.

14. Oppenheim AV, Schafer RW, Buck JR. Discrete Time Signal Processing. London: Pearson; 1999. 870 p.

15. Benitez D, Gaydecki PA, Zaidi A, et al. The use of the Hilbert transform in ECG signal analysis. Comput Biol Med. 2001;31(5):399-406.

16. Hastie T, Tibshirani R, Friedman J. The Elements of Statistical Learning. Newyork: Springer; 2009. 337-387 p.

17. Gibbons JD, Chakraborti S. Nonparametric Statistical Inference [Internet]. Newyork: Marcel Dekker; 2003. 672 p. 